\title{
Fermented drinks of HippophaeRhamnoides as a way of innovative development of agriculture in Altai region
}

\author{
Evgeny Rozhnov ${ }^{1,2}$, Marina Schkolnikova ${ }^{1}$, Vladimir Popov ${ }^{1}$ \\ ${ }^{1}$ Ural State University of Economics, 620144 Yekaterinburg, Russian Federation \\ ${ }^{2}$ Biysk technical Institute(branch campus) "Polzunov Altai State Technical University", 659305 \\ Biysk, Russian Federation
}

\begin{abstract}
One of the technological innovations nowadays is the development of industrial processing of agricultural raw material, manufacturing process leading to lower risks - «Low/No/Reduced» and «Better-for-you». This may be done by light processing of raw materials and use of traditional technologies, i.e. fermentation of craft beverages by natural symbiotic cultures Oryzamycesindichiand Medusomycesgisevi (kombucha). The recent years saw a worldwide interest to a horticultural crop called Hippophaerhamnoides (sea-buckthorn) as it is eco-friendly and healthful. Its fruits have a special smell and taste, are reach in carotene, carotenoids, tocopherols, ascorbic acids, polyphenols. The composition is the reason why it is used for foodstuff, i.e. pastry, dairy products, unclarified juice. Production of beverages fermented by natural symbiotic cultures and local orchard products is a new direction in Russia. The supplies are limited which means that the research is relevant. The aim of the research is to study the possibility of usage ofjuice of Hippophaerhamnoides fruits in the new technology of soft drinks production fermented by Oryzamycesindichi and Medusomycesgisevi. A single nutritious substrate for Oryzamycesindichi and Medusomycesgisevi symbiotic cultures cultivation was proved impossible to be produced. Still the possibility to replace polyphenols of the Medusomycesgisevi by those of the sea-buckthorn juice must be analyzed.Sea-buckthorn juice added in a culture medium fermented by Oryzamycesindichi and Medusomycesgisevi symbiotic cultures increases biosynthetic activity of acidforming bacteria. This tangibly improves organoleptic properties of the fermented soft beverages produced. Besides, the technology of Oryzamycesindichi and Medusomycesgisevi production presupposes no clarification which makes the process of the Hippophaerhamnoides use significantly easier. The research helped to identify that the sea-buckthorn juice used in a must fermented by Medusomycesgisevi has many advantages. Namely more comprehensive utilization of carbohydrate, a higher level of reduction of organic acids, and decrease of the time of technological process ( 24 hours less).
\end{abstract}




\section{Introduction}

Sea-buckthornis atree and shrubbery foodplant used for alimentary, therapeutic, and decorative purposes. The culture differs by the ability to synthesize oil in the pulp and the skin, to be polymorphic, eco-friendly, easy to keep, frost and drought resistant $[1,2]$. The results of the work of the Lisavenko Research Institute of Horticultures of Siberia enabled to create industrial plantations of sea-buckthorn in Altai Region. A most significant for the industry feature of the culture is the convenient fruit ripening period - from the mid-August until the end of October for the early and the late ones resp. [3-6].

The foodstuff containing sea-buckthorn is becoming demanded and is treated healthy in many countries. This may be juice, candies, jellies, jams, alcoholic and soft beverages, dairy products [7].

The fruits have a special smell and taste that may be characterized as acid, sour, astringent, and not sweet. The total amount and/or proportion of sugars and acids is a crucial characteristic that regulates the taste of the fruits and ways of their processing $[8,9]$. Besides, the sea-buckthorn has a complex composition influencing the production of fermented strong and soft beverages[10-12].

While unclarified products are being produced, i.a. juice or fruit nectar, small parts of the oil join to big flake-like structures and float on beverages, which diminishes its sensorial qualities. Yet the smallest suspend oil drops complicate the filtration of the seabuckthorn juice to be further clarified [13].

A key innovation in food manufacturing is development of industrial processing of agricultural raw material. The process leads to lower risks - «Low/No/Reduced» and «Better-for-you». Firstly, these directions presuppose a light processing of natural product, first, as modern consumers avoid consuming harshly processed products. Secondly, traditional processes and technologies, such as fermentation, appear to be favored by Russian and foreign producers. They are simpler and more easily accepted by consumers because of the naturality as compared to "artificial" modern technologies. Thirdly, a popular trend is craft food and beverages. It is a chance for the manufacturers selling "home-made" products by old recipes to enter the market. Craft beverages fermented by natural symbiotic cultures Oryzamycesindichi[14-16]andMedusomycesgisevi (kombucha[17, 18]) may be ones of the products.

\section{Purpose of the study}

Beverages of natural symbiotic cultures are quite demanded in the world market, but in Russia they are new, as proved by the limited supply. Thus, the development of scientific bases for fruit raw material use, i.e. for Hippophaerhamnoides, in technologies of traditional fermentation of beverages is relevant for production and processing of horticultural crops. So, the aim of the research is to study the possibility to use the juice of Hippophaerhamnoides fruits to produce soft drinks fermented by Oryzamycesindichi and Medusomycesgisevi.

\section{Objects of the study}

The objects of the study were samples of fermented soft beverages with directly squeezed juice of sea-buckthorn added and Oryzamycesindichi and Medusomycesgisevi symbiotic cultures used (fig. 1). 


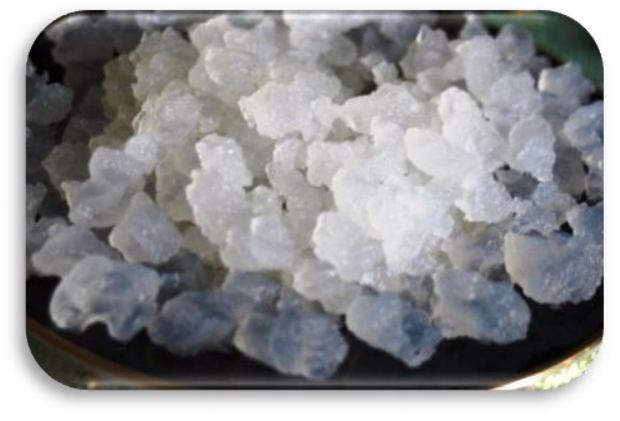

Oryzamycesindichi

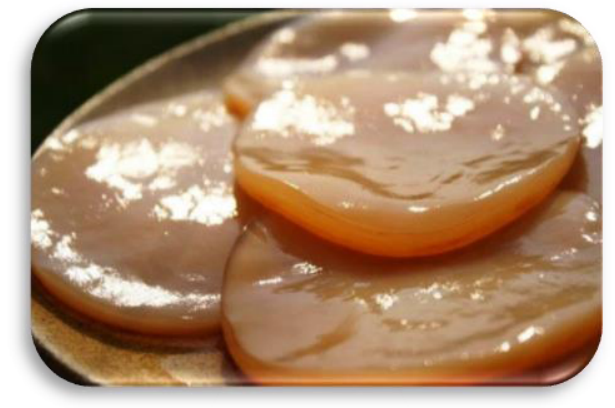

Medusomycesgisevi

Fig. 1.The symbiotic cultures

The directly squeezed sea-buckthorn juice was made of the berries of a variety called "Chuyskaya". The berries were cut and treated by a pectolytic enzyme Rapidase CR (dosage $20 \mathrm{ml} /$ tof raw material, treatment time 3 hours, temperature $50{ }^{\circ} \mathrm{C}$ ), thenpressed in the expeller. The further organoleptic assessment showed that the juice has a sour- sweet and astringent taste. The smell corresponds to the one of the fresh berries of the variety. The composition is presented in the table 1 .

Table 1.Composition of the directly squeezed sea-buckthorn juice ( $n=3, M \pm m$ )

\begin{tabular}{|c|c|c|c|c|}
\hline \multirow{2}{*}{ Sample } & \multicolumn{4}{|c|}{ Mass content, \% } \\
\cline { 2 - 5 } & $\begin{array}{c}\text { Organic acids on } \\
\text { a malic acid basis }\end{array}$ & sugars & Dry basis & $\begin{array}{c}\text { Polyphenolic } \\
\text { substances }\end{array}$ \\
\hline Juice & $1,18 \pm 0,04$ & $8,12 \pm 0,08$ & $13,2 \pm 0,1$ & $0,125 \pm 0,011$ \\
\hline
\end{tabular}

The juice-containing substrate was fermented by Oryzamycesindichi as follows. The substrates made as formulated were centrifugated to remove suspended particulate matter (SPM). Then they were pasteurized during an hour at $65 \pm 2{ }^{\circ} \mathrm{C}$ to eliminate foreign microflora affecting the activity of the product and organoleptic, physical and chemical properties of the beverage. First, the culture Oryzamycesindichi $5 \%$ wt was inserted and, second, the specieswere thermostated for 5 days in the hot air oven at $25 \pm 1{ }^{\circ} \mathrm{C}$ to cultivate the substrate. The fermented broth was withdrawn once a day to analyze its physical and chemical properties. The cultivated fermented beverage was separated from the zoogloea through decantation and was further put to refrigerate at $5-7{ }^{\circ} \mathrm{C}$ for the next experiments. The zoogloea was constantly washed with distilled water and returned to fermentation.

The juice-containing substrate was fermented by Medusomycesgisevi as follows. The substrates made as formulated were centrifugated to remove suspended particulate matter (SPM). Then they were pasteurized during an hour at $65 \pm 2{ }^{\circ} \mathrm{C}$ to eliminate foreign microflora affecting the activity of the product and organoleptic, physical and chemical properties of the beverage. First, the culture Medusomycesgisevi 5\% wt was inserted and, second, the species were thermostated for 5 days in the hot air oven at $25 \pm 1{ }^{\circ} \mathrm{C}$ to cultivate the substrate. The fermented broth was withdrawn once a day to analyze its physical and chemical properties. The cultivated fermented beverage was separated from the zoogloea through decantation and was further put to refrigerate at $5-7{ }^{\circ} \mathrm{C}$ for the next experiments. The lower layer of the zoogloea was constantly removed and the zoogloea was washed with distilled water and returned to fermentation.

The control samples were beverages without the sea-buckthorn juice made likewise. 


\section{Materials and methods}

The physical and chemical properties of the sea-buckthorn juice and the samples of the beverages were identified by standard methods. The fermented beverages were titrated with 1 mol of sodium hydroxide as per GOST 6687.4-86 to discover the acid number. The mass content of sugars was determined by the Bertrand copper-reduction method as per GOST 8756.13-87. The mass content of dry basis was found by the aerometric method as per GOST 6687.2-90. The volume concentration of ethyl hydroxide was learnt by the distillation method as per GOST 6687.7-88. The mass of polyphenolic substances was measured by the colorimetry with the Folin-Ciocalteu reagent (FCR) [19]. The absorptivity was detected with the wavelength at $610 \mathrm{~nm}$ in the Shimadzu UV-1800 Doble-Beam Scanning Spectrophotometer. Individual organic acids of the samples were analyzed by the capillary electrophoresis (CE) in the system "Kapel' 105M" (Russia, LLC "Lumex"). The conditions were as follows: sampling at $150 \mathrm{mbar} \times \mathrm{sec}$, voltage at minus $20 \mathrm{kv}$, thermostat temperature at $20^{\circ} \mathrm{C}$. The acids were detected by an indirect method of ultraviolet absorption at $254 \mathrm{~nm}$. Organic acids were separated by a buffer solution of $10 \mathrm{mmol}$ of benzoic acid, $9 \mathrm{mmol}$ of diethanolamine, $0,5 \mathrm{mmol}$ ofcetrimonium bromide, $0,5 \mathrm{mmol}$ of ethylenediaminetetraacetic acid and capillaries of $75 \mu \mathrm{mol}$ in dimeter and $60 \mathrm{~cm}$ in length. The collection of data and processing of electropherograms were done in the software "Elphoran" of the "Kapel' 105M". The peaks were identified in quality and in quantity using standard calibration solutions.

\section{Results and discussion}

The literature data showed that composition of culture media for Oryzamycesindichi and Medusomycesgisevi symbiotic cultures cultivation differs. Thus, the culture Oryzamycesindichi needs digestible carbohydrates only to reproduce. The source of nitrogenous matters necessary for the symbiont's normal vital activity, described in the research [20], was a small quantity of raisin. It may be replaced by fruit and berry juice [21] which would allow to arrange complex industrial processing of Oryzamycesindichi using sea-buckthorn juice.

However, the culture Medusomycesgisevi requires big quantity of polyphenols, i.a. the ones of the tea liquor, to develop.

Thus, the single nutritious substrate for the Oryzamycesindichi and Medusomycesgisevi symbiotic cultures cultivation is provedimpossible to be produced. Still the possibility to replace polyphenols of the Medusomycesgisevi by those of the sea-buckthorn juice must be analyzed.

The table 2 shows the composition of musts for cultivation of the symbiotic cultures for the beverages with the final acidity $5 \mathrm{~g} / \mathrm{dm} 3 \mathrm{max}$. The analysis made demonstrates that the acidity of the beverages rise generally to $1,5-1,8 \mathrm{~g} / \mathrm{dm} 3$ when fermented $[20,21]$.

Table 2.Composition of samples of must for fermentation ofbeverages (\%)

\begin{tabular}{|l|c|c|}
\hline \multicolumn{1}{|c|}{ Raw material } & Oryzamycesindichi & Medusomycesgisevi \\
\hline Directly squeezed sea-buckthorn juice & 25,0 & 25,0 \\
\hline Sucrose & 10,0 & 10,0 \\
\hline Tea liquor* & - & 2,25 \\
\hline Drinking water & to 100,0 & to 100,0 \\
\hline *Quantity of polyphenols in the strong liquor $-0,215 \pm 0,007 \%$ \\
\hline
\end{tabular}


An aqueous extract of polyphenols of green tea leaves as a component of the must is added to cultivate the kombucha. This eliminates the unattractive for consumers grayish orange and brown color of the fermented teal leaves. The composition of the must samples made for Oryzamycesindichi and Medusomycesgisevi cultivation is presented in the table 3.

Table 3.Composition of samples of musts ( $n=3, M \pm m)$

\begin{tabular}{|l|c|c|c|}
\hline \multirow{2}{*}{ Sample } & \multicolumn{3}{|c|}{ Mass content, \% } \\
\cline { 2 - 4 } & $\begin{array}{c}\text { Organic acids } \\
\text { on a malic acid } \\
\text { basis }\end{array}$ & Sugars & $\begin{array}{c}\text { Polyphenolic } \\
\text { substances }\end{array}$ \\
\hline $\begin{array}{l}\text { Oryzamycesindichiand } \\
\text { sea-buckthorn juice }\end{array}$ & $0,32 \pm 0,02$ & $10,0 \pm 0,1$ & $0,031 \pm 0,003$ \\
\hline $\begin{array}{l}\text { Medusomycesgiseviand } \\
\text { sea-buckthorn juice }\end{array}$ & $0,31 \pm 0,02$ & $10,0 \pm 0,1$ & $0,052 \pm 0,004$ \\
\hline
\end{tabular}

The fig. 2 demonstrates the dynamic of organic acids, sugar utilization, and ethyl alcohol accumulated in 5 days in the process of Oryzamycesindichi and Medusomycesgisevi cultivation.

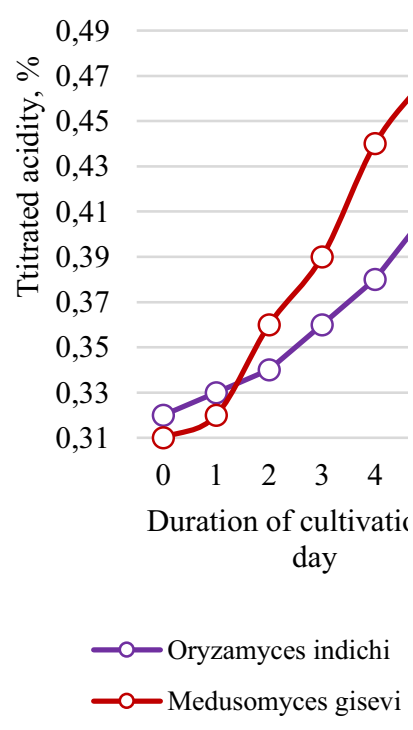

a)

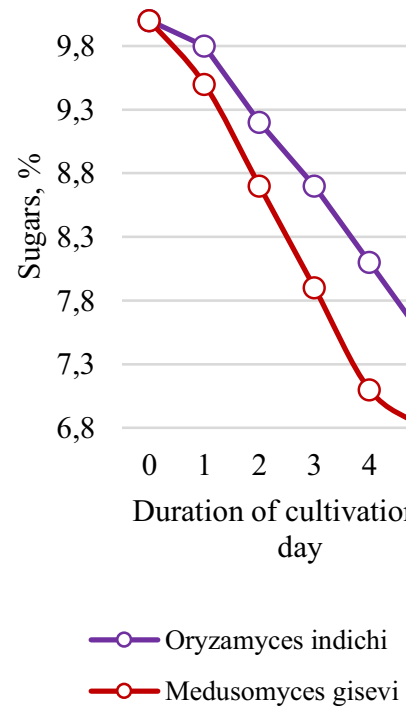

b)

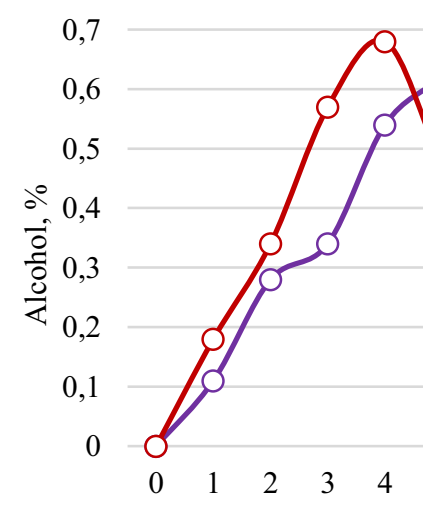

Duration of cultivation peı

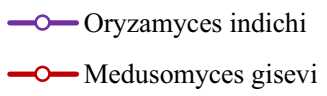

c)

Fig. 2.Dynamic of organic acids (a), sugar (b), and ethyl alcohol (c) accumulated in 5 days of Oryzamycesindichi and Medusomycesgisevi cultivation

The fig. 2 shows that the fermentation of juice-containing substrates using the symbiotic cultures is likewise. However, utilization of nutritious carbohydrates of the must with Medusomycesgisevi cultivation is larger. This results in a more intensive accumulation of ethanol in the medium and in higher concentration of titrated acids. The concentration is due tofavorable conditions for reproduction of Acetobacteraceti and Acetobacterxylinum, prone to biosynthesize ethanoic acid. The results obtained on identification of organic acids in the beverages by the CE method is the proof (fig. 3). 


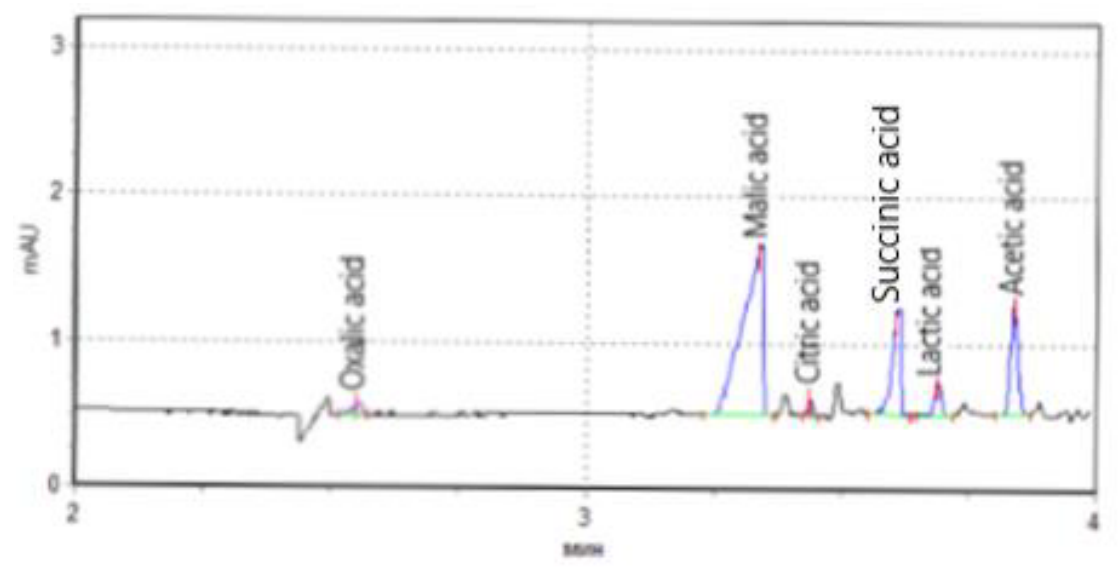

Fig. 3. Typical electropherogram of the beverage with Medusomycesgisevi cultivated for 5 days

Table 4 (results of the CE) demonstrates that the dominant organic acid in the fermented drinks, with the cultures cultivated for 5 days on the substrates, is the malic one. This can be explained by the fact that the malic acid was integrated in the beverage along with the raw material. Its quantity suffered almost no changes during cultivation. The increment in quantity of succinic, lactic, and ethanoic acids can stem from biosynthesis of the microorganisms of the Oryzamycesindichi and Medusomycesgisevi symbiotic cultures.

Table 4.Dynamic of synthesized organic acids in cultivation of the Oryzamycesindichi and Medusomycesgisevi cultures $(n=3, M \pm m)$

\begin{tabular}{|c|c|c|c|c|c|}
\hline \multirow{2}{*}{$\begin{array}{c}\text { Organic } \\
\text { acid }\end{array}$} & \multicolumn{5}{|c|}{$\begin{array}{c}\text { Concentration of the organic acids during the fermenta- } \\
\text { tion, mg/dm }\end{array}$} \\
\cline { 2 - 6 } & Day 1 & Day 2 & Day 3 & Day 4 & Day 5 \\
\cline { 2 - 6 } & \multicolumn{5}{|c|}{ Control ofOryzamycesindichi } \\
\hline Succinic & $111,6 \pm 0,9$ & $134,7 \pm 0,6$ & $167,2 \pm 0,4$ & $179,3 \pm 0,5$ & $193,2 \pm 0,4$ \\
\hline Lactic & - & $13,6 \pm 0,3$ & $24,7 \pm 0,2$ & $47,1 \pm 0,3$ & $89,3 \pm 0,4$ \\
\hline Ethanolic & - & - & $39,4 \pm 0,3$ & $147,9 \pm 0,5$ & $354,6 \pm 0,6$ \\
\hline & \multicolumn{5}{|c|}{ Oryzamycesindichi with sea-buckthorn juice } \\
\hline Succinic & $118,6 \pm 0,8$ & $139,7 \pm 0,3$ & $178,2 \pm 0,8$ & $192,4 \pm 0,3$ & $213,4 \pm 0,6$ \\
\hline Lactic & - & $17,5 \pm 0,2$ & $36,9 \pm 0,2$ & $67,4 \pm 0,2$ & $102,6 \pm 0,3$ \\
\hline Ethanolic & - & $27,9 \pm 1,1$ & $47,8 \pm 0,8$ & $173,2 \pm 1,1$ & $492,4 \pm 1,3$ \\
\hline & \multicolumn{5}{|c|}{ Control of Medusomycesgisevi } \\
\hline Succinic & $98,7 \pm 0,7$ & $164,3 \pm 0,9$ & $204,1 \pm 0,9$ & $274,2 \pm 1,3$ & $318,3 \pm 2,6$ \\
\hline Lactic & - & - & \multicolumn{5}{c|}{$11,4 \pm 1,7$} & $24,7 \pm 1,0$ \\
\hline Ethanolic & $108,2 \pm 1,3$ & $179,6 \pm 2,4$ & $267,2 \pm 2,9$ & $381,9 \pm 3,2$ & $505,8 \pm 4,6$ \\
\hline & \multicolumn{5}{|c|}{ Medusomycesgiseviwith sea-buckthorn juice } \\
\hline Succinic & $93,7 \pm 0,5$ & $174,3 \pm 1,2$ & $227,3 \pm 1,6$ & $264,3 \pm 0,9$ & $293,6 \pm 2,2$ \\
\hline Lactic & - & $16,4 \pm 0,3$ & $34,7 \pm 1,1$ & $72,3 \pm 1,0$ & $125,8 \pm 2,0$ \\
\hline Ethanolic & $134,7 \pm 2,2$ & $204,7 \pm 2,8$ & $316,4 \pm 2,4$ & $483,5 \pm 3,2$ & $762,3 \pm 3,7$ \\
\hline
\end{tabular}

The results obtained can confirm that sea-buckthorn juice added in the culture medium, with fermentation of Oryzamycesindichi and Medusomycesgisevi symbiotic cultures, results in biosynthetic activity of acid-forming bacteria. This also effects organoleptic 
properties of soft fermented beverages. However, the sharp quantitative increase of synthesized ethanoic acid by Medusomycesgisevi leads to organoleptic properties improved within 4 days of fermentation. A steep increase in biosynthesis of organic acids was not registered for fermentation of the control sample and the sample with sea-buckthorn juice by Oryzamycesindichi. The duration of fermentation remained the same.

The fig. 4 and 5 are profile charts of organoleptic properties of the beverages obtained by fermentation of must samples by the Oryzamycesindichi and Medusomycesgisevi cultures for 5 days compared to the control data.

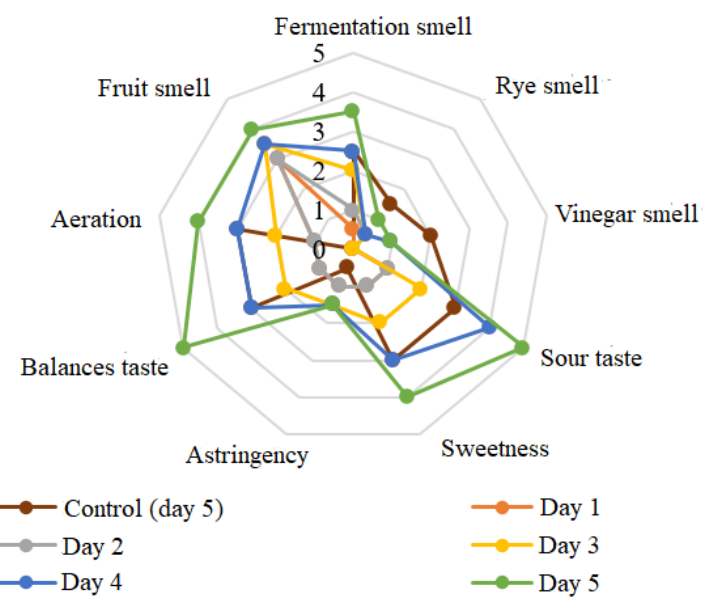

Fig. 4.Profile chart of organoleptic properties of cultivated for 5 days Oryzamycesindichi with seabuckthorn

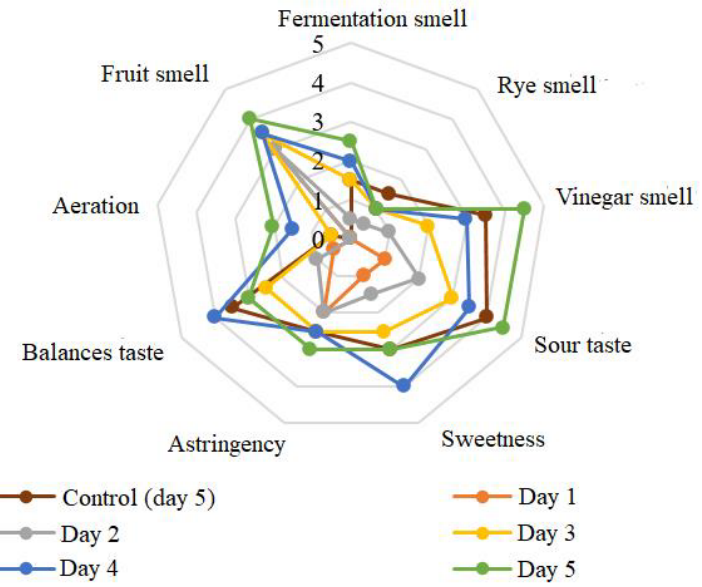

Fig. 5.Profile chart of organoleptic properties of cultivated for 5 days Medusomycesgisevi with seabuckthorn

Hence, sea-buckthorn juice used in the beverage with Oryzamycesindichi improves organoleptic properties, making the smell and taste more present and masking the rye flavor, as well as the strong smell of ethanoic acid, present in the control sample. The beverage remains greyish light-yellow without any impurities. Foamability and short-lasting release 
of carbon dioxide, while being poured, are the remarkable properties too. The taste is sweet-and-sour, refreshing and nice.

The assessment of organoleptic properties of the control and experimental samples of kombucha with sea-buckthorn showed that the sea-buckthorn juice changed the taste and the smell of the beverage. Kombucha with sea-buckthorn beverage is grayish yellow without any impurities. The taste of the berries is sweet-and-sour, astringent. The smell is complex, comprising fermentation smell, nuances of the variety raw material, and the typical vinegar smell.

\section{Conclusions}

In the course of research it was stated that Hippophaerhamnoides used as raw material to produce soft beverages fermented by traditional symbiotic cultures, i.e. Oryzamycesindichi and Medusomycesgisevi, was proved to improve organoleptic properties of beverages. However, the technologies of production of Oryzamycesindichi and Medusomycesgisevi presuppose no clarification which makes the process of Hippophaerhamnoides use significantly easier. The research helped to identify that the sea-buckthorn juice used in the must fermented by Medusomycesgisevi results in many advantages. They are more comprehensive utilization of carbohydrate, a higher level of re-duction of organic acids, and decrease in time of technological process ( 24 hours less than for the traditional one).

Thus, the possibility to implement Hippophaerhamnoides in technologies of fermentation of soft beverages not traditional for the Russian Federation was studied. Improved organoleptic properties of the samples obtained demonstrate the potential of the suggested craft technologies, with Oryzamycesindichi and Medusomycesgisevi, should be used for industrial purpose.

\section{References}

1. Yu. A. Koshelev, L.D. Ageeva, E.S. Batashov, V.P. Sevodin, E.D. Rozhnov, N.I. Kuleshova, Sea buckthorn, 410 (Publishing house of Polzunov Altai State Technical, Biysk, 2015)

2. I.K. Dugarova, G.Ts. Tsybikova, I.T. Alexandrova, IzvestiyaVuzov. Prikladnaya Khimiyai Biotekhnologiya, 6 (3), 128 (2016)

3. A. Raffo, F. Paoletti, M. Antonelli, Eur Food Res Technol, 219, 360 (2004)

4. S.C. Andersson, M.E. Olsson, E. Johansson,K. Rumpunen, J. Agric. Food Chem., 57 (1), 250 (2009)

5. S. Arif, S.D. Ahmed, A.H. Shah, L. Hassan, S.I. Awan, A. Hamid, F. Batool, Pak. J. Bot., 42 (5), 3561 (2010)

6. X. Gao, M. Ohlander, N. Jeppsson, L. Björk,V. Trajkovski, J. Agric. Food Chem., 48 (5), 148 (2000)

7. E. Christaki, Food and Public Health, 2 (3), 69 (2012)

8. A. Kårlund, K. Hanhineva, M. Lehtonen, R.O. Karjalainen,M. Sandell, J. of Agricultural and Food Chemistry, 63 (3), 1010 (2015)

9. O. Laaksonen, L. Mäkilä, R. Tahvonen, H. Kallio, B. Yang, Food Chemistry, 138 (4), 2421 (2013) 
10. B. Negi,G. Dey, World Academy of Science, Engineering and Technology, 30, 99 (2009)

11. S.L. Wang, L.P. Liu, L.X. Jiao,M.T. Fan, Int. J. of Food Properties, 14, 776 (2011)

12. S.L. Wang, L.X. Jiao, Y.H. Li, M.T. Fan, Int. J. of Food Properties, 15, 1381 (2010)

13. R. Arimboor, V.V. Venugopalan, K. Sarinkumar, C. Arumughan, R.C. Sawhney, J. of the Science of Food and Agriculture, 86 (14), (2006)

14. J. Stadie, A. Gulitz, M.A. Ehrmann, R.F. Vogel, Food Microbiology, 35 (2), 92 (2013)

15. D. Laureys, L.D. Vuyst, Applied and Environmental Microbiology, 80 (8), 2564 (2014)

16. M.S. Muneer Alsayadi, A.J. Yaser, M. Belarbi, F.Z. Sabri, J. of Microbiology, Biotechnology and Food Sciences, 2 (6), 2444 (2013)

17. I. Vina, P. Semjonovs, R. Linde,I. Denina,J. of Medicinal Food, 17 (2), 179 (2014)

18. J.M. Leal, L.V. Suarez, R. Jayabalan, J.H. Oros,A. Escalante-Aburto, J. of Food, 16 (1), 390 (2018)

19. A. Blainski,G.C. Lopes,J.C. Palazzo de Mello, Molecules, 18 (6), 6852 (2013)

20. Yu.S. Zintsova, E.D. Rozhnov, M.N. Shkolnikova, Technology and the study of merchandise of innovative foodstuffs, 3 (32), 37-42 (2015)

21. Yu.S. Zintsova, M.N. Shkolnikova, E.D. Rozhnov, Tovaroved prodovolstvennykh tovarov, 7, 12 (2015) 